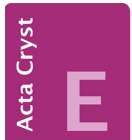
COMMUNICATIONS

ISSN 2056-9890

\section{Crystal structure of $\mathrm{N}$-(4-chlorophenyl)- benzothioamide}

\section{Ganlin Zhao}

Changsha Environmental Protection College, Changsha 410004, People's Republic of China. *Correspondence e-mail: hbxygcx2011@126.com

Received 20 April 2015; accepted 23 April 2015

Edited by H. Stoeckli-Evans, University of Neuchâtel, Switzerland

The title compound, $\mathrm{C}_{13} \mathrm{H}_{10} \mathrm{ClNS}$, exhibits a trans conformation with regard to the axis of the $\mathrm{C}-\mathrm{N}$ bond. The benzene and phenyl rings are inclined to one another by $85.06(8)^{\circ}$. In the crystal, molecules are linked by $\mathrm{N}-\mathrm{H} \cdots \mathrm{S}=\mathrm{C}$ hydrogen bonds, forming chains along [001].

Keywords: crystal structure; benzothioamide; $\mathrm{N}-\mathrm{H}$...S hydrogen bonding..

CCDC reference: 1061304

\section{Related literature}

For hydrogen bonding of amides, see: Taylor et al. (1984); Leiserowitz \& Schmidt (1969). For the preparation and for the use of thioamides as intermediates in chemical transformations, see: Li et al. (2012, 2015). For related structures, see: Omondi et al. (2012); Nagasawa et al. (2014).<smiles>S=C(Nc1ccc(Cl)cc1)c1ccccc1</smiles>

\section{Experimental}

\subsection{Crystal data}

$\mathrm{C}_{13} \mathrm{H}_{10} \mathrm{ClNS}$

$$
M_{r}=247.73
$$

Monoclinic, $P 2_{b} / c$
$a=11.943(2) \AA$
$b=12.689(3) \AA$
$c=7.9764(16) \AA$
$\beta=109.30(3)^{\circ}$
$V=1140.9(4) \AA^{3}$

\subsection{Data collection}

Rigaku Saturn CCD area-detector diffractometer

Absorption correction: multi-scan (CrystalClear; Rigaku/MSC, 2005)

$T_{\min }=0.901, T_{\max }=0.944$

\subsection{Refinement}

$R\left[F^{2}>2 \sigma\left(F^{2}\right)\right]=0.028$

$w R\left(F^{2}\right)=0.077$

$S=1.05$

2010 reflections

150 parameters

1 restraint

Table 1

Hydrogen-bond geometry $\left(\AA,{ }^{\circ}\right)$.

\begin{tabular}{lllll}
\hline$D-\mathrm{H} \cdots A$ & $D-\mathrm{H}$ & $\mathrm{H} \cdots A$ & $D \cdots A$ & $D-\mathrm{H} \cdots A$ \\
\hline $\mathrm{N} 1-\mathrm{H} 1 \cdots \mathrm{S} 1^{\mathrm{i}}$ & $0.89(1)$ & $2.49(1)$ & $3.346(15)$ & $163(1)$ \\
\hline Symmetry code: (i) $x,-y+\frac{3}{2}, z-\frac{1}{2}$. & &
\end{tabular}

Data collection: CrystalClear (Rigaku/MSC, 2005); cell refinement: CrystalClear; data reduction: CrystalClear; program(s) used to solve structure: SHELXS97 (Sheldrick, 2008); program(s) used to refine structure: SHELXL97 (Sheldrick, 2008); molecular graphics: SHELXTL (Sheldrick, 2008); software used to prepare material for publication: SHELXL97.

Supporting information for this paper is available from the IUCr electronic archives (Reference: SU5123).

\section{References}

Leiserowitz, L. \& Schmidt, G. M. (1969). J. Chem. Soc. A, pp. 2372-2382. Li, J. S., Cheng, C., Zhang, X. R., Li, Z. W., Cai, F. F., Xue, Y. \& Liu, W. D. (2012). Chin. J. Chem. 30, 1687-1689.

Li, J. S., Xue, Y., Li, P. Y., Li, Z. W., Lu, C. H., Liu, W. D., Pang, H. L., Liu, D. H., Lin, M. S., Luo, B. B. \& Jiang, W. (2015). Res. Chem. Intermed. 41, 2235-2247.

Nagasawa, M., Sasanuma, Y. \& Masu, H. (2014). Acta Cryst. E70, o639.

Omondi, B. \& Levendis, D. C. (2012). Acta Cryst. E68, o2604.

Rigaku/MSC (2005). CrystalClear. Rigaku Corporation, Tokyo, Japan.

Sheldrick, G. M. (2008). Acta Cryst. A64, 112-122.

Taylor, R., Kennard, O. \& Versichel, W. (1984). Acta Cryst. B40, 280-288. 


\section{supporting information}

Acta Cryst. (2015). E71, o353 [https://doi.org/10.1107/S2056989015008075]

\section{Crystal structure of $\mathrm{N}$-(4-chlorophenyl) benzothioamide}

\section{Ganlin Zhao}

\section{S1. Comment}

Thioamides are extensively used as extractant for heavy metals in environmental chemistry, as intermediate for important chemical transformations (Li et al., 2015), and also used to replace the amide bonds as isosteres. It's well known that amide units can be connected by a $\mathrm{N}-\mathrm{H} \cdots \mathrm{O}=\mathrm{C}$ hydrogen bonds (Taylor et al., 1984; Leiserowitz \& Schmidt, 1969), and also the structures of some thioamides have been documented (Omondi et al., 2012; Nagasawa et al., 2014). Herein, we report the crystal structure of the title compound (I).

Figure 1 has shows the molecular structure of the title compound, whose thioamide unit adopts a trans conformation around the central $\mathrm{C}-\mathrm{N}$ bond. The $\mathrm{C}=\mathrm{S}$ double bond is deviated from its connected phenyl ring [torsion angles:

$\left.\mathrm{S} 1 / \mathrm{C} 7 / \mathrm{C} 8 / \mathrm{C} 9-145.34(13)^{\circ}, \mathrm{S} 1 / \mathrm{C} 7 / \mathrm{C} 8 / \mathrm{C} 1333.62(19)^{\circ}\right]$. The benzene and phenyl rings are inclined to one another by $85.06(8)^{\circ}$.

In the crystal, molecules are linked via $\mathrm{N}-\mathrm{H} \cdots \mathrm{S}=\mathrm{C}$ hydrogen bonds, forming chains along the $c$ axis direction (Table 1 and Fig. 2).

\section{S2. Experimental}

The title compound was prepared from the Beckmann rearrangement from its corresponding ketoximes following a published procedure (Li et al., 2012). It was isolated by flash chromatography and yellow block-like crystal of the title compound were obtained via natural evaporation from the diluent.

\section{S3. Refinement}

The thioamide $\mathrm{N}-\mathrm{H}$ atom was located in a difference Fourier map and freely refined. The $\mathrm{C}$-bound $\mathrm{H}$ atoms were included in calculated positions and refined as riding atoms: $\mathrm{C}-\mathrm{H}=0.93 \AA$ with $U_{\text {iso }}(\mathrm{H})=1.2 U_{\text {eq }}(\mathrm{C})$.

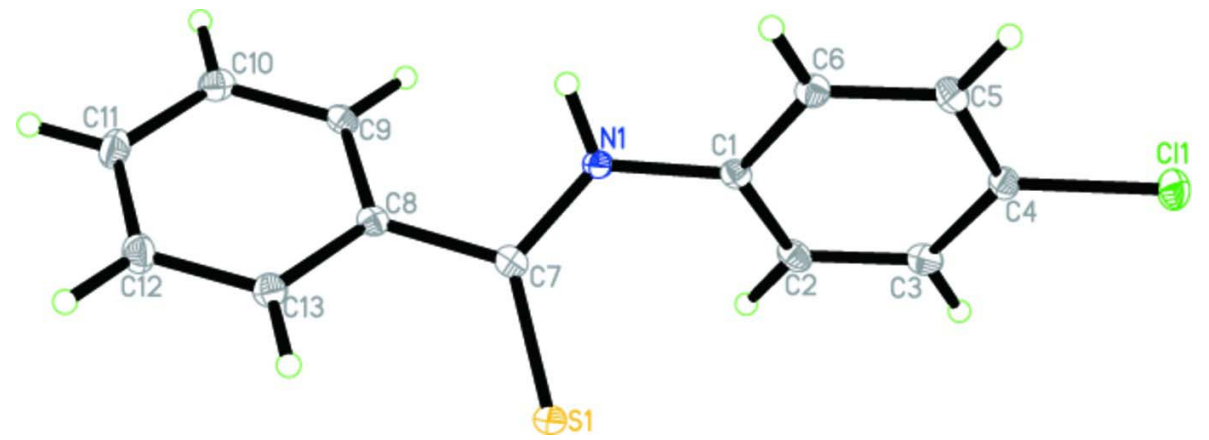

Figure 1

The molecular structure of the title compound, with atom labelling. Displacement ellipsoids are drawn at the 30\% probability level. 


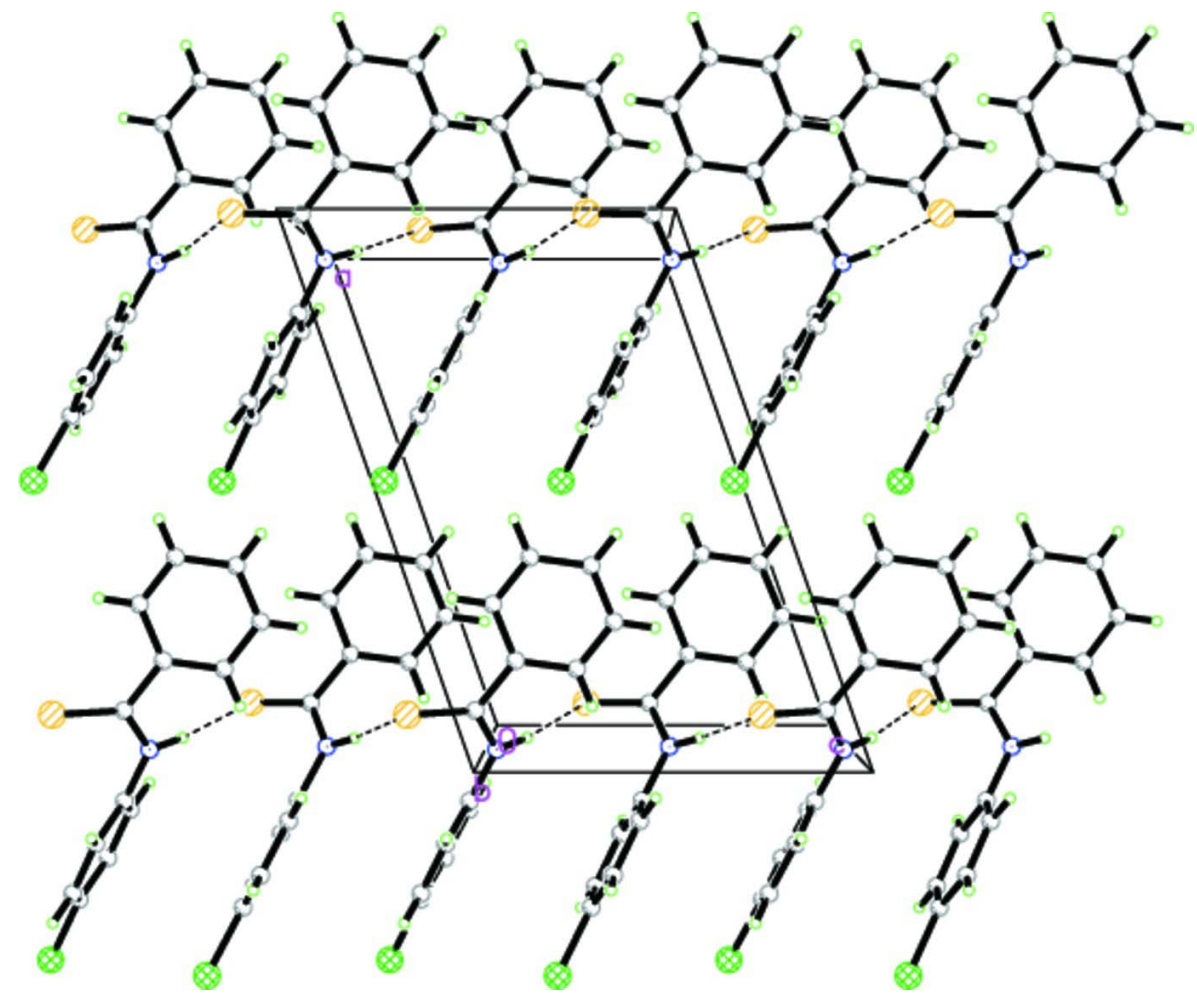

Figure 2

A view along the $b$ axis of the crystal packing of the title compound. The $\mathrm{N}-\mathrm{H} \cdots \mathrm{S}$ hydrogen bonds are shown as dashed lines (see Table 1 for details).

\section{$N$-(4-Chlorophenyl)benzothioamide}

Crystal data

$\mathrm{C}_{13} \mathrm{H}_{10} \mathrm{ClNS}$

$M_{r}=247.73$

Monoclinic, $P 2{ }_{1} / c$

Hall symbol: -P $2 \mathrm{ybc}$

$a=11.943(2) \AA$

$b=12.689$ (3) $\AA$

$c=7.9764$ (16) $\AA$

$\beta=109.30(3)^{\circ}$

$V=1140.9(4) \AA^{3}$

$Z=4$

\section{Data collection}

Rigaku Saturn CCD area-detector diffractometer

Radiation source: rotating anode

Confocal monochromator

Detector resolution: 7.31 pixels $\mathrm{mm}^{-1}$ $\omega$ and $\varphi$ scans

Absorption correction: multi-scan

(CrystalClear; Rigaku/MSC, 2005)

$T_{\min }=0.901, T_{\max }=0.944$
$F(000)=512$

$D_{\mathrm{x}}=1.442 \mathrm{Mg} \mathrm{m}^{-3}$

Mo $K \alpha$ radiation, $\lambda=0.71073 \AA$

Cell parameters from 3499 reflections

$\theta=1.8-27.9^{\circ}$

$\mu=0.49 \mathrm{~mm}^{-1}$

$T=113 \mathrm{~K}$

Block, yellow

$0.22 \times 0.20 \times 0.12 \mathrm{~mm}$

7517 measured reflections

2010 independent reflections

1701 reflections with $I>2 \sigma(I)$

$R_{\text {int }}=0.030$

$\theta_{\max }=25.0^{\circ}, \theta_{\min }=2.4^{\circ}$

$h=-11 \rightarrow 14$

$k=-15 \rightarrow 15$

$l=-9 \rightarrow 9$ 


\section{Refinement}

Refinement on $F^{2}$

Least-squares matrix: full

$R\left[F^{2}>2 \sigma\left(F^{2}\right)\right]=0.028$

$w R\left(F^{2}\right)=0.077$

$S=1.05$

2010 reflections

150 parameters

1 restraint

Primary atom site location: structure-invariant direct methods

Secondary atom site location: difference Fourier map
Hydrogen site location: inferred from neighbouring sites

$\mathrm{H}$ atoms treated by a mixture of independent and constrained refinement

$w=1 /\left[\sigma^{2}\left(F_{\mathrm{o}}^{2}\right)+(0.0477 P)^{2}+0.1743 P\right]$ where $P=\left(F_{\mathrm{o}}{ }^{2}+2 F_{\mathrm{c}}{ }^{2}\right) / 3$

$(\Delta / \sigma)_{\max }=0.002$

$\Delta \rho_{\max }=0.22 \mathrm{e} \AA^{-3}$

$\Delta \rho_{\min }=-0.18$ e $\AA^{-3}$

Extinction correction: SHELXL97 (Sheldrick, 2008), $\mathrm{Fc}^{*}=\mathrm{kFc}\left[1+0.001 \mathrm{xFc}^{2} \lambda^{3} / \sin (2 \theta)\right]^{-1 / 4}$

Extinction coefficient: 0.050 (4)

\section{Special details}

Geometry. All e.s.d.'s (except the e.s.d. in the dihedral angle between two 1.s. planes) are estimated using the full covariance matrix. The cell e.s.d.'s are taken into account individually in the estimation of e.s.d.'s in distances, angles and torsion angles; correlations between e.s.d.'s in cell parameters are only used when they are defined by crystal symmetry. An approximate (isotropic) treatment of cell e.s.d.'s is used for estimating e.s.d.'s involving 1.s. planes.

Refinement. Refinement of $F^{2}$ against ALL reflections. The weighted $R$-factor $w R$ and goodness of fit $S$ are based on $F^{2}$, conventional $R$-factors $R$ are based on $F$, with $F$ set to zero for negative $F^{2}$. The threshold expression of $F^{2}>\sigma\left(F^{2}\right)$ is used only for calculating $R$-factors(gt) etc. and is not relevant to the choice of reflections for refinement. $R$-factors based on $F^{2}$ are statistically about twice as large as those based on $F$, and $R$ - factors based on ALL data will be even larger.

Fractional atomic coordinates and isotropic or equivalent isotropic displacement parameters $\left(\AA^{2}\right)$

\begin{tabular}{lllll}
\hline & $x$ & $y$ & $z$ & $U_{\text {iso }} * U_{\text {eq }}$ \\
\hline S1 & $0.94504(4)$ & $0.58973(3)$ & $1.20150(5)$ & $0.01919(16)$ \\
C11 & $1.47747(4)$ & $0.84240(4)$ & $1.54329(6)$ & $0.02760(17)$ \\
N1 & $1.02619(11)$ & $0.71403(10)$ & $0.99722(17)$ & $0.0160(3)$ \\
C1 & $1.13474(13)$ & $0.74209(12)$ & $1.1311(2)$ & $0.0159(3)$ \\
C2 & $1.21244(14)$ & $0.66609(13)$ & $1.2321(2)$ & $0.0201(4)$ \\
H2 & 1.1936 & 0.5949 & 1.2147 & $0.024^{*}$ \\
C3 & $1.31777(14)$ & $0.69722(13)$ & $1.3583(2)$ & $0.0218(4)$ \\
H3 & 1.3698 & 0.6470 & 1.4264 & $0.026^{*}$ \\
C4 & $1.34551(14)$ & $0.80302(13)$ & $1.3828(2)$ & $0.0186(4)$ \\
C5 & $1.26984(15)$ & $0.87901(13)$ & $1.2816(2)$ & $0.0219(4)$ \\
H5 & 1.2895 & 0.9501 & 1.2982 & $0.026^{*}$ \\
C6 & $1.16448(15)$ & $0.84782(13)$ & $1.1550(2)$ & $0.0194(4)$ \\
H6 & 1.1134 & 0.8982 & 1.0857 & $0.023^{*}$ \\
C7 & $0.94159(14)$ & $0.64895(12)$ & $1.0128(2)$ & $0.0159(4)$ \\
C8 & $0.83987(14)$ & $0.63239(12)$ & $0.8467(2)$ & $0.0161(4)$ \\
C9 & $0.85648(15)$ & $0.62942(12)$ & $0.6821(2)$ & $0.0182(4)$ \\
H9 & 0.9320 & 0.6389 & 0.6751 & $0.022^{*}$ \\
C10 & $0.76076(16)$ & $0.61237(13)$ & $0.5280(2)$ & $0.0226(4)$ \\
H10 & 0.7725 & 0.6106 & 0.4184 & $0.027^{*}$ \\
C11 & $0.64826(16)$ & $0.59810(13)$ & $0.5376(2)$ & $0.0249(4)$ \\
H11 & 0.5842 & 0.5868 & 0.4347 & $0.030^{*}$ \\
C12 & $0.63130(15)$ & $0.60079(13)$ & $0.7015(2)$ & $0.0251(4)$ \\
H12 & 0.5557 & 0.5910 & 0.7082 & $0.030^{*}$ \\
& & & &
\end{tabular}




$\begin{array}{lllll}\text { C13 } & 0.72593(14) & 0.61784(12) & 0.8545(2) & 0.0201(4) \\ \text { H13 } & 0.7137 & 0.6197 & 0.9637 & 0.024^{*} \\ \text { H1 } & 1.0058(16) & 0.7552(13) & 0.9013(17) & 0.030(5)^{*}\end{array}$

Atomic displacement parameters $\left(\AA^{2}\right)$

\begin{tabular}{lllllll}
\hline & $U^{11}$ & $U^{22}$ & $U^{33}$ & $U^{12}$ & $U^{13}$ & $U^{23}$ \\
\hline S1 & $0.0205(2)$ & $0.0192(2)$ & $0.0179(3)$ & $-0.00251(17)$ & $0.00650(18)$ & $0.00248(16)$ \\
C11 & $0.0174(2)$ & $0.0353(3)$ & $0.0268(3)$ & $-0.00442(18)$ & $0.00281(18)$ & $-0.00048(18)$ \\
N1 & $0.0177(7)$ & $0.0151(7)$ & $0.0155(7)$ & $-0.0010(6)$ & $0.0057(6)$ & $0.0010(6)$ \\
C1 & $0.0158(8)$ & $0.0181(8)$ & $0.0164(8)$ & $-0.0003(7)$ & $0.0090(7)$ & $-0.0010(6)$ \\
C2 & $0.0187(9)$ & $0.0154(8)$ & $0.0280(9)$ & $0.0004(7)$ & $0.0102(7)$ & $0.0010(7)$ \\
C3 & $0.0170(8)$ & $0.0215(9)$ & $0.0279(10)$ & $0.0042(7)$ & $0.0089(7)$ & $0.0066(7)$ \\
C4 & $0.0138(8)$ & $0.0245(9)$ & $0.0186(8)$ & $-0.0014(7)$ & $0.0070(7)$ & $-0.0008(7)$ \\
C5 & $0.0231(9)$ & $0.0177(8)$ & $0.0249(9)$ & $-0.0026(7)$ & $0.0081(8)$ & $-0.0024(7)$ \\
C6 & $0.0192(9)$ & $0.0175(8)$ & $0.0211(9)$ & $0.0029(7)$ & $0.0063(7)$ & $0.0016(6)$ \\
C7 & $0.0173(8)$ & $0.0118(7)$ & $0.0210(9)$ & $0.0028(6)$ & $0.0096(7)$ & $-0.0021(6)$ \\
C8 & $0.0186(8)$ & $0.0098(7)$ & $0.0201(8)$ & $0.0007(6)$ & $0.0065(7)$ & $-0.0005(6)$ \\
C9 & $0.0202(9)$ & $0.0133(8)$ & $0.0223(9)$ & $-0.0018(7)$ & $0.0085(7)$ & $0.0000(7)$ \\
C10 & $0.0300(10)$ & $0.0194(8)$ & $0.0186(9)$ & $-0.0017(8)$ & $0.0081(8)$ & $-0.0018(7)$ \\
C11 & $0.0234(9)$ & $0.0257(9)$ & $0.0202(9)$ & $-0.0010(8)$ & $0.0001(7)$ & $-0.0017(7)$ \\
C12 & $0.0171(9)$ & $0.0280(9)$ & $0.0285(10)$ & $0.0010(8)$ & $0.0052(7)$ & $0.0009(8)$ \\
C13 & $0.0192(9)$ & $0.0210(9)$ & $0.0212(9)$ & $0.0019(7)$ & $0.0080(7)$ & $0.0011(7)$ \\
& & & & & & \\
\hline
\end{tabular}

Geometric parameters $\left(A,{ }^{\circ}\right)$

\begin{tabular}{llll}
\hline $\mathrm{S} 1-\mathrm{C} 7$ & $1.6705(16)$ & $\mathrm{C} 6-\mathrm{H} 6$ & 0.9300 \\
$\mathrm{C} 11-\mathrm{C} 4$ & $1.7430(17)$ & $\mathrm{C} 7-\mathrm{C} 8$ & $1.487(2)$ \\
$\mathrm{N} 1-\mathrm{C} 7$ & $1.342(2)$ & $\mathrm{C} 8-\mathrm{C} 9$ & $1.391(2)$ \\
$\mathrm{N} 1-\mathrm{C} 1$ & $1.426(2)$ & $\mathrm{C} 8-\mathrm{C} 13$ & $1.395(2)$ \\
$\mathrm{N} 1-\mathrm{H} 1$ & $0.891(9)$ & $\mathrm{C} 9-\mathrm{C} 10$ & $1.392(2)$ \\
$\mathrm{C} 1-\mathrm{C} 6$ & $1.385(2)$ & $\mathrm{C} 9-\mathrm{H} 9$ & 0.9300 \\
$\mathrm{C} 1-\mathrm{C} 2$ & $1.395(2)$ & $\mathrm{C} 10-\mathrm{C} 11$ & $1.383(2)$ \\
$\mathrm{C} 2-\mathrm{C} 3$ & $1.384(2)$ & $\mathrm{C} 10-\mathrm{H} 10$ & 0.9300 \\
$\mathrm{C} 2-\mathrm{H} 2$ & 0.9300 & $\mathrm{C} 11-\mathrm{C} 12$ & $1.388(3)$ \\
$\mathrm{C} 3-\mathrm{C} 4$ & $1.381(2)$ & $\mathrm{C} 11-\mathrm{H} 11$ & 0.9300 \\
$\mathrm{C} 3-\mathrm{H} 3$ & 0.9300 & $\mathrm{C} 12-\mathrm{C} 13$ & $1.379(2)$ \\
$\mathrm{C} 4-\mathrm{C} 5$ & $1.384(2)$ & $\mathrm{C} 12-\mathrm{H} 12$ & 0.9300 \\
$\mathrm{C} 5-\mathrm{C} 6$ & $1.385(2)$ & $\mathrm{C} 13-\mathrm{H} 13$ & 0.9300 \\
$\mathrm{C} 5-\mathrm{H} 5$ & 0.9300 & & $115.00(13)$ \\
& & & $124.40(13)$ \\
$\mathrm{C} 7-\mathrm{N} 1-\mathrm{C} 1$ & $127.60(13)$ & $\mathrm{N} 1-\mathrm{C} 7-\mathrm{C} 8$ & $120.59(12)$ \\
$\mathrm{C} 7-\mathrm{N} 1-\mathrm{H} 1$ & $116.1(12)$ & $\mathrm{N} 1-\mathrm{C} 7-\mathrm{S} 1$ & $118.95(15)$ \\
$\mathrm{C} 1-\mathrm{N} 1-\mathrm{H} 1$ & $114.8(12)$ & $\mathrm{C} 8-\mathrm{C} 7-\mathrm{S} 1$ & $121.01(15)$ \\
$\mathrm{C} 6-\mathrm{C} 1-\mathrm{C} 2$ & $119.92(15)$ & $\mathrm{C} 9-\mathrm{C} 8-\mathrm{C} 13$ & $120.03(15)$ \\
$\mathrm{C} 6-\mathrm{C} 1-\mathrm{N} 1$ & $118.25(14)$ & $\mathrm{C} 9-\mathrm{C} 8-\mathrm{C} 7$ & $120.42(16)$ \\
$\mathrm{C} 2-\mathrm{C} 1-\mathrm{N} 1$ & $121.77(14)$ & $\mathrm{C} 13-\mathrm{C} 8-\mathrm{C} 7$ & \\
$\mathrm{C} 3-\mathrm{C} 2-\mathrm{C} 1$ & $119.60(15)$ & $\mathrm{C} 8-\mathrm{C} 9-\mathrm{C} 10$ &
\end{tabular}




\begin{tabular}{|c|c|c|c|}
\hline $\mathrm{C} 3-\mathrm{C} 2-\mathrm{H} 2$ & 120.2 & $\mathrm{C} 8-\mathrm{C} 9-\mathrm{H} 9$ & 119.8 \\
\hline $\mathrm{C} 1-\mathrm{C} 2-\mathrm{H} 2$ & 120.2 & $\mathrm{C} 10-\mathrm{C} 9-\mathrm{H} 9$ & 119.8 \\
\hline $\mathrm{C} 4-\mathrm{C} 3-\mathrm{C} 2$ & $119.90(15)$ & $\mathrm{C} 11-\mathrm{C} 10-\mathrm{C} 9$ & $120.03(16)$ \\
\hline $\mathrm{C} 4-\mathrm{C} 3-\mathrm{H} 3$ & 120.0 & $\mathrm{C} 11-\mathrm{C} 10-\mathrm{H} 10$ & 120.0 \\
\hline $\mathrm{C} 2-\mathrm{C} 3-\mathrm{H} 3$ & 120.0 & $\mathrm{C} 9-\mathrm{C} 10-\mathrm{H} 10$ & 120.0 \\
\hline $\mathrm{C} 3-\mathrm{C} 4-\mathrm{C} 5$ & $120.96(15)$ & $\mathrm{C} 10-\mathrm{C} 11-\mathrm{C} 12$ & $119.76(16)$ \\
\hline $\mathrm{C} 3-\mathrm{C} 4-\mathrm{Cl} 1$ & $119.94(13)$ & $\mathrm{C} 10-\mathrm{C} 11-\mathrm{H} 11$ & 120.1 \\
\hline $\mathrm{C} 5-\mathrm{C} 4-\mathrm{Cl1}$ & $119.09(13)$ & $\mathrm{C} 12-\mathrm{C} 11-\mathrm{H} 11$ & 120.1 \\
\hline $\mathrm{C} 4-\mathrm{C} 5-\mathrm{C} 6$ & $119.13(15)$ & $\mathrm{C} 13-\mathrm{C} 12-\mathrm{C} 11$ & $120.34(16)$ \\
\hline $\mathrm{C} 4-\mathrm{C} 5-\mathrm{H} 5$ & 120.4 & $\mathrm{C} 13-\mathrm{C} 12-\mathrm{H} 12$ & 119.8 \\
\hline $\mathrm{C} 6-\mathrm{C} 5-\mathrm{H} 5$ & 120.4 & $\mathrm{C} 11-\mathrm{C} 12-\mathrm{H} 12$ & 119.8 \\
\hline $\mathrm{C} 1-\mathrm{C} 6-\mathrm{C} 5$ & $120.46(15)$ & $\mathrm{C} 12-\mathrm{C} 13-\mathrm{C} 8$ & $120.50(16)$ \\
\hline $\mathrm{C} 1-\mathrm{C} 6-\mathrm{H} 6$ & 119.8 & $\mathrm{C} 12-\mathrm{C} 13-\mathrm{H} 13$ & 119.7 \\
\hline $\mathrm{C} 5-\mathrm{C} 6-\mathrm{H} 6$ & 119.8 & $\mathrm{C} 8-\mathrm{C} 13-\mathrm{H} 13$ & 119.7 \\
\hline $\mathrm{C} 7-\mathrm{N} 1-\mathrm{C} 1-\mathrm{C} 6$ & $-131.37(16)$ & $\mathrm{C} 1-\mathrm{N} 1-\mathrm{C} 7-\mathrm{S} 1$ & $2.6(2)$ \\
\hline $\mathrm{C} 7-\mathrm{N} 1-\mathrm{C} 1-\mathrm{C} 2$ & $51.1(2)$ & $\mathrm{N} 1-\mathrm{C} 7-\mathrm{C} 8-\mathrm{C} 9$ & $35.2(2)$ \\
\hline $\mathrm{C} 6-\mathrm{C} 1-\mathrm{C} 2-\mathrm{C} 3$ & $1.3(2)$ & $\mathrm{S} 1-\mathrm{C} 7-\mathrm{C} 8-\mathrm{C} 9$ & $-145.34(13)$ \\
\hline $\mathrm{N} 1-\mathrm{C} 1-\mathrm{C} 2-\mathrm{C} 3$ & $178.80(14)$ & $\mathrm{N} 1-\mathrm{C} 7-\mathrm{C} 8-\mathrm{C} 13$ & $-145.86(15)$ \\
\hline $\mathrm{C} 1-\mathrm{C} 2-\mathrm{C} 3-\mathrm{C} 4$ & $-0.3(2)$ & $\mathrm{S} 1-\mathrm{C} 7-\mathrm{C} 8-\mathrm{C} 13$ & $33.62(19)$ \\
\hline $\mathrm{C} 2-\mathrm{C} 3-\mathrm{C} 4-\mathrm{C} 5$ & $-0.7(2)$ & $\mathrm{C} 13-\mathrm{C} 8-\mathrm{C} 9-\mathrm{C} 10$ & $0.1(2)$ \\
\hline $\mathrm{C} 2-\mathrm{C} 3-\mathrm{C} 4-\mathrm{Cl} 1$ & $179.35(12)$ & $\mathrm{C} 7-\mathrm{C} 8-\mathrm{C} 9-\mathrm{C} 10$ & $179.08(14)$ \\
\hline $\mathrm{C} 3-\mathrm{C} 4-\mathrm{C} 5-\mathrm{C} 6$ & $0.5(2)$ & $\mathrm{C} 8-\mathrm{C} 9-\mathrm{C} 10-\mathrm{C} 11$ & $-0.1(2)$ \\
\hline $\mathrm{C} 11-\mathrm{C} 4-\mathrm{C} 5-\mathrm{C} 6$ & $-179.46(12)$ & $\mathrm{C} 9-\mathrm{C} 10-\mathrm{C} 11-\mathrm{C} 12$ & $0.0(2)$ \\
\hline $\mathrm{C} 2-\mathrm{C} 1-\mathrm{C} 6-\mathrm{C} 5$ & $-1.5(2)$ & $\mathrm{C} 10-\mathrm{C} 11-\mathrm{C} 12-\mathrm{C} 13$ & $0.2(3)$ \\
\hline $\mathrm{N} 1-\mathrm{C} 1-\mathrm{C} 6-\mathrm{C} 5$ & $-179.01(14)$ & $\mathrm{C} 11-\mathrm{C} 12-\mathrm{C} 13-\mathrm{C} 8$ & $-0.1(2)$ \\
\hline $\mathrm{C} 4-\mathrm{C} 5-\mathrm{C} 6-\mathrm{C} 1$ & $0.5(2)$ & $\mathrm{C} 9-\mathrm{C} 8-\mathrm{C} 13-\mathrm{C} 12$ & $0.0(2)$ \\
\hline $\mathrm{C} 1-\mathrm{N} 1-\mathrm{C} 7-\mathrm{C} 8$ & $-177.96(13)$ & $\mathrm{C} 7-\mathrm{C} 8-\mathrm{C} 13-\mathrm{C} 12$ & $-178.98(14)$ \\
\hline
\end{tabular}

Hydrogen-bond geometry $\left(\AA,{ }^{\circ}\right)$

\begin{tabular}{lllll}
\hline$D-\mathrm{H} \cdots A$ & $D-\mathrm{H}$ & $\mathrm{H} \cdots A$ & $D \cdots A$ & $D-\mathrm{H} \cdots A$ \\
\hline $\mathrm{N} 1-\mathrm{H} 1 \cdots \mathrm{S} 1^{\mathrm{i}}$ & $0.89(1)$ & $2.49(1)$ & $3.346(15)$ & $163(1)$ \\
\hline
\end{tabular}

Symmetry code: (i) $x,-y+3 / 2, z-1 / 2$. 